\title{
In-Situ Control of Defect Dynamics By Ellipsometry During Ion Implantation - Evolution of Disorder and Cavity Structure in Single-Crystalline Ge During Implantation of Sb lons
}

Tivadar Lohner

MTA Centre for Energy Research

Attila Németh

MTA Wigner Research Centre for Physics

Zsolt Zolnai

MTA Centre for Energy Research

Benjamin Kalas

MTA Centre for Energy Research

Alekszej Romanenko

MTA Centre for Energy Research

Nguyen Khánh

MTA Centre for Energy Research

Edit Szilágyi

MTA Wigner Research Centre for Physics

\section{Endre Kótai}

MTA Wigner Research Centre for Physics

\section{Emil Agócs}

MTA Centre for Energy Research

\section{Zsolt Tóth}

University of Szeged

Judit Budai

ELI-HU Research and Development Non-Profit

Peter Petrik ( $\sim$ petrik.peter@ek-cer.hu )

MTA Centre for Energy Research

Miklos Fried

MTA Centre for Energy Research

Isvtán Bársony

MTA Centre for Energy Research

József Gyulai

MTA Centre for Energy Research 


\section{Research Article}

Keywords: dynamics, defect, evolution, crystalline

DOl: https://doi.org/10.21203/rs.3.rs-132556/v1

License: (c) (i) This work is licensed under a Creative Commons Attribution 4.0 International License. Read Full License 


\section{Abstract}

Ion implantation has been a key technology in microelectronics and generally, for the controlled surface modification of materials for tribology, biocompatibility, corrosion resistance and many more. In this work in-situ spectroscopic ellipsometry was used for accurately tracking and on-line evaluating the accumulation of voids and damage in crystalline Ge during implantation of $200-\mathrm{keV} \mathrm{Sb}^{+}$ions at a total fluence of $10^{16} \mathrm{~cm}^{-2}$ using an ion flux of $2.1 \times 10^{12} \mathrm{~cm}^{-2} \mathrm{~s}^{-1}$. The phases of damage accumulation were identified using unique optical multi-layer models describing the layer structure and composition. The formation of initial partial disorder was followed by complete amorphization and void formation occurring at the fluence of $1 \times 10^{15} \mathrm{~cm}^{-2}$, reaching a high volume fraction of voids and a layer thickness of $\approx 200 \mathrm{~nm}$ by the end of the process. This agrees with numerical simulations and results of complementary measurements including ion beam analysis and electron microscopy. The developed insitu method for controlling the dynamics of structural damage accumulation is a versatile ionimplantation tool for avoiding adverse void formation and for controlled evolution of subsurface nanocavities or cellular surface texture alike.

\section{Highlights}

- Integration of spectroscopic ellipsometry in an ion implantation chamber

- Models for the depth distribution of damage and void formation

- In-situ characterization of the structural changes by a temporal resolution of $3 \mathrm{~s}$

- Determination of the dynamics of cellular structure formation by ion implantation

- Mechanism of void formation during ion implantation

\section{Introduction}

The interest in Ge has been increasing in recent years due to the broad range of potential applications from photonics ${ }^{1}$ to microelectronics. It has been utilized in high-speed photodetectors that are compatible with Si microtechnology, providing a cost-effective alternative to III-V semiconductors ${ }^{2}$. Ge and its alloys (primarily with $\mathrm{Si}$ ) are also used as Bragg reflectors ${ }^{3}$, photodiodes ${ }^{2}$, materials of controlled optical properties (especially in the infrared wavelength range) ${ }^{4}$, as well as in band gap ${ }^{5}$ and refractive index ${ }^{6}$ engineering. Although the size-dependent optical properties of Ge were measured earlier ${ }^{7}$ than those of $\mathrm{Si}^{8}$, the attention of researchers has turned only recently to Ge nanocrystals ${ }^{9,10}$ (NCs). Some remarkable features of $\mathrm{Ge}$, including the wide tunable spectral range, stronger quantum confinement (QC) effects, compound applications ${ }^{11,12}$, biocompatibility, and electrochemical stability looked very attractive. For instance, the low effective masses and the large exciton radius of about $24 \mathrm{~nm}$ in Ge allows to control QC effects on a larger length scale as compared to, e.g., Si, with exciton radius of only about $4.5 \mathrm{~nm}^{13}$. 
Device structures are usually prepared by subsequent deposition/implantation ${ }^{14,15}$ and lithography processes. Ion implantation is used for the controlled doping or modification of crystallinity ${ }^{16}$, Due to the fact that both energy and fluence of the ion beam can be precisely controlled, the material properties obtained by ion implantation are highly reproducible. Furthermore, the temporal and spatial variation of disorder can be influenced by the choice of the implanted element ${ }^{17}$ and the ion flux, i.e., the ion current ${ }^{18}$. The profile of the implanted elements and disorder have different depth distributions that can be controlled by the energy ${ }^{19,20}$ and direction of the implantation ${ }^{21}$.

Depending on which material property was in the focus of the study, various methods of characterization of the implanted structure had been applied. Structural measurements are usually conducted by highresolution scanning ${ }^{22}$ or transmission ${ }^{6}$ electron microscopy, HR-SEM and HR-TEM, respectively. Both surface topography ${ }^{22}$ and in-depth microstructure ${ }^{6}$ have been imaged, revealing a sponge-like structure with voids in many cases, e.g. for $50-300-k e V$ self-ion bombardment ${ }^{22}$. Structural properties such as the size of crystals or the degree of amorphization can also be determined by optical methods $6,7,9,23$. Rutherford backscattering spectrometry (RBS) allows to reveal the degree of crystallinity, crystal structure (when combined with channeling) and the depth distribution of elements. Its combination with spectroscopic ellipsometry (SE) is a powerful way of complementary depth profiling, primarily in crystalline semiconductors ${ }^{11,24-26}$. SE provides also thickness reference values for density determination of thin layers analyzed by RBS ${ }^{25,27}$. The combination of different in-situ and ex-situ characterization methods with SE is very popular. Recently, in an Auger electron spectroscopy study the complementarity of both methods was analyzed ${ }^{28}$. Besides determination of the optical properties ${ }^{14,15,23,29}$, frequently in SiGe alloys $s^{6,11,12}$, used in optics or optoelectronics, optical methods are also capable of indirectly determining material properties, such as the crystallinity ${ }^{19}$, disorder ${ }^{30}$ or crystal size ${ }^{7}$. Finally, there are numerous electrical characterization methods for the quality control of Ge and Ge NC-based devices. ${ }^{31}$

Spectroscopic ellipsometry (SE) ${ }^{32}$ is one of the most sensitive methods to detect the change of crystallinity in semiconductors. SE determines the complex dielectric function of $\varepsilon=\varepsilon_{1}+i \varepsilon_{2}, \varepsilon_{1}$ and $\varepsilon_{2}$ being the real and imaginary parts, respectively, with a precision of $\approx 10^{-4}$. Since $\varepsilon_{2}$ is proportional to the joint density of electron states, the method is very sensitive to the change of long-range order in the lattice, i.e. to the crystallinity of the material ${ }^{33}$. Due to the large amount of spectroscopic data, complex models can be built with parameters that describe the formation of structures in depth ${ }^{26,34}$. Owing to the non-destructive nature of ellipsometry, in-situ applications were demonstrated by many authors ${ }^{35}$. Albeit many papers discussed ex-situ ellipsometric characterization of Ge structures ${ }^{14}$, in-situ measurement of ion implantation has only been studied by $\mathrm{Hu}$ et al. upon cleaning and layer removal by $\mathrm{He}^{36}$ and $\mathrm{Ar}^{37}$ ions. In their work Hu et al. characterized the etching and damage evolution in the top $10 \mathrm{~nm}$ of native and oxidized Si wafers during the implantation with low energy (300-1200 eV) $\mathrm{Ar}^{37}$ ions by in-situ SE. The main reason of the in-situ study was in both cases to measure the effect of surface modification in the vacuum before the appearance of any contamination from the air. There was no attempt to strive for 
a high temporal resolution. In our case, however, as the initial phase of amorphization occurs within a couple of seconds, it can only be followed and analyzed if complete spectra can quickly be recorded.

A number of studies are dealing with the optical and structural characterization of Ge prepared by a wide range of methods, ${ }^{12,14,15,23}$ but only a few papers discuss the optical and structural characterization of disordered Ge layers obtained by ion bombardment. ${ }^{19}$ Aspnes and Studna irradiated single-crystalline $\mathrm{Ge}$ (c-Ge) surfaces using $\mathrm{Ne}$ and Ar ions at an energy of $1 \mathrm{keV}$. The SE measurements on ultra-thin (less than $10 \mathrm{~nm}$ ) ion implantation-amorphized $\mathrm{Ge}$ (i-a-Ge) layers made possible to determine the dielectric function of i-a-Ge with more than $10 \%$ uncertainty. Broad damage distributions were realized by multiple-step and energy implantations, which are useful for the determination of optical references. ${ }^{23}$ It was also proven that the implantation of heavy elements results in quick total amorphization up to the surface of the sample ${ }^{38,39}$, but without a honeycomb void formation and with less void than found for Ge in the present study.

The implantation of Ge by light elements in c-Ge was used by several authors to create a dense and uniform i-a-Ge structure in a controlled way ${ }^{19,40}$. In a previous work we used double-step and -energy Alimplantation to create an amorphous reference for the effective medium approximation (EMA) method for characterization of damage profiles in $\mathrm{Ge}^{23}$. When implanting heavy elements in $\mathrm{Ge}$ (self-implantation or heavier than $\mathrm{Ge}$ ), void formation occurs, which was not found in $\mathrm{Si}^{41} \mathrm{CdTe}^{39}$ and $\mathrm{SiC}^{42}$ Above a certain fluence in c-Ge bombarded by heavy ions, a peculiar cellular structure develops ${ }^{6,19,20,22,40,43-47}$. This provides an opportunity for a self-organized pattern generation controlled by the ion implantation parameters for which in-situ ellipsometry can offer a real-time feedback. The damage and void formation in Ge were investigated by several groups, but no in-situ measurements have been performed. All studies so far were carried out after the implantation process, investigating only the final structure after reorganization and relaxation processes. To follow the dynamics of the complex process of amorphization and void formation, we mounted an ellipsometer onto an ion implantation chamber, and recorded spectroscopic data in the visible-near ultraviolet wavelength range recording complete spectra at a time resolution of $3 \mathrm{~s}$ during the implantation of $200-\mathrm{keV} \mathrm{Sb}$ ions into c-Ge. The authors of this work have knowledge of only a single study ${ }^{37}$ in which the layer structure has optically been measured during the implantation process. To our best knowledge, however, there is no publication available on in-situ observation of the effect of ion implantation into Ge during the bombardment process preformed in vacuum.

The aim of this work was to follow the evolution of disorder and morphology changes induced by ion bombardment in c-Ge. In our previous work ${ }^{23}$, the complex dielectric function of i-a-Ge was determined by SE in a broad wavelength range. This result was used as a basic component during the evaluation of the present in-situ measurements. We fitted the parameters of a complex optical model with a temporal resolution of $3 \mathrm{~s}$, to follow the evolution of the different parameters, such as sublayer thicknesses and volumetric ratios of c-Ge, i-a-Ge and voids in the sublayer. 


\section{Results}

\section{Integration of SE into a vacuum chamber}

For real-time in-situ SE measurements an M-88 spectroscopic ellipsometer (J.A. Woollam Co. Inc.) with a rotating analyzer was mounted on a high vacuum chamber of the Heavy lon Cascade Implanter of the Hungarian lon-beam Physics Platform at the Institute for Particle and Nuclear Physics, Wigner Research Centre for Physics, Budapest. The vacuum chamber shown in Fig. 1 is equipped with high-quality entrance and exit windows to minimize the deviation in the polarization state of the measuring light caused by birefringence. The angle of incidence is fixed at $75^{\circ}$. The spectral range from 367 to $746 \mathrm{~nm}$ was measured in 72 spectral points in every 3 seconds, simultaneously. The spectra in the UV and IR regions were limited by the windows and the instrument, respectively. The alignment of the sample was realized by a 4-axis precision goniometer to maximize the reflected intensity, followed by an offset calibration. The sample holder includes a Faraday cup that reduces the typical value of uncertainty of the ion current measurement from $5-10-1 \% .{ }^{48}$

\section{In-situ SE measurement during ion implantation}

The evolution of the measured pseudo refractive index $(n)$ and extinction coefficient $(k)$ spectra is plotted in Fig. 2. The ion beam was turned on at about $60 \mathrm{~s}$ (a sharp feature in the recorded spectra close to the zero fluence position), and it was turned off at a fluence of $1 \cdot 10^{16} \mathrm{~cm}^{-2}$ (after $4800 \mathrm{~s}$ ). The continuous evolution of all the spectra is evident from Fig. 2, underlining the need for the spectroscopic measurement. Note the rapid changes at the start and at the fluences of $\approx 2 \cdot 10^{15} \mathrm{~cm}^{-2}$. The optical penetration depth (OPD) shows these characteristic features for the red part of the spectrum (above the wavelength of approximately $600 \mathrm{~nm}$ ), as the penetration depth abruptly decreases at the beginning of the irradiation due to amorphization, and increases again in the above mentioned range between 1.9 and $2.5 \cdot 10^{15} \mathrm{~cm}^{-2}$ due to void formation, as it will be shown in the detailed optical model analysis below.

\section{Structural characterization}

The scanning electron micrograph in Fig. 3A shows a peculiar cellular structure on the surface of the 200$\mathrm{keV} \mathrm{Sb}{ }^{+}$-implanted Ge sample. The surface contains cavities (black appearance) with horizontal dimensions of about $10 \mathrm{~nm}$ to $100 \mathrm{~nm}$. Holland et al. compared this structure to honeycombs ${ }^{40}$. Moreover, very similar surface structure has been also observed after $3 \mathrm{MeV} \mathrm{I}^{+}$irradiation of $\mathrm{Ge}^{46}$. Calculations by the SRIM (Stopping and Range of lons in Matter ${ }^{49}$ ) software shows a peak concentration of a few percent for the $\mathrm{Sb}$ atom peak concentration (without taking into account the effect of sputtering and void formation). The damaged range of approximately $100 \mathrm{~nm}$ was revealed by the vacancy distribution (Fig. 3B). The peak damage is located at about $40 \mathrm{~nm}$ depth and extends to end of range of the implanted ions. Note that a displacement threshold energy of $30 \mathrm{eV}^{50}$ for Ge atoms has been considered in SRIM simulations. 


\section{Discussion}

\section{Phases of damaged layer formation}

The features of the measured ellipsometric signals (Figs. 2 and 4) as well as the evolution of the structure can be subdivided into 4 regions: (I) a baseline before switching on the ion beam, (II) damage accumulation, (III) rapid void formation, (IV) slow change of damage and voids, revealed by the increasing $\Psi$ signal in Fig. 4.

The baseline of the ellipsometric angles was taken from region I and it was used to determine the dielectric function of the c-Ge substrate. From the numerous possible parameterizations of the dielectric function ${ }^{33}$, the dispersion of the c-Ge wafer was described using the Johs-Herzinger generalized critical point model ${ }^{51}$. Only the oscillator parameters of $\mathrm{Ge}$ transitions at $2.1,2.3$, and $3.4 \mathrm{eV}$ were fitted in a c$\mathrm{Ge} / \mathrm{GeO}_{2}$ model. Subsequently, these parameters were fixed during the evaluation of the spectra measured during the ion implantation.

In regions II-IV a uniform two-layer model was used with components of c-Ge, Ge amorphized by implantation ( $\mathrm{i}-\mathrm{a}-\mathrm{Ge}$ ) and voids. Note that for the $\mathrm{i}-\mathrm{a}-\mathrm{Ge}$ component the parameters were taken from our previous work on $\mathrm{Ge}$ amorphized by $\mathrm{Al}$ ion implantation ${ }^{23}$. The effective dielectric function of each layer was calculated using the Bruggeman effective medium approximation $\left(E M A^{52}\right)$. The initial dielectric function of $\mathrm{i}-\mathrm{a}-\mathrm{Ge}^{23}$ was parameterized by the Tauc-Lorentz dispersion model, in which only the energy position of the single Lorentz oscillator was fitted. A deviation in the dielectric function of i-a-Ge makes sense, taking into account that amorphous semiconductors may have different optical properties depending on the preparation conditions. ${ }^{12,14,15,23,34,53,54}$

The thickness of the layers rapidly changes in region III, partly because of the increasing penetration depth of the implanted ions due to the void formation. The void fraction in the top layer accounts for the roughness ${ }^{52}$ as well. In the last phase of structure formation (Region IV) both the void fraction and the thickness of the bottom layer increases monotonically.

Figure 5 shows measured and fitted $\Psi$ and $\Delta$ spectra for selected characteristic temporal points of the structure formation. The graphs reveal that the curves vary significantly in terms of time and spectral features. Note that the repeatability of the measurements of both $\psi$ and $\Delta$ is $\approx 0.2^{\circ}$, a small fraction of the width of the plotted lines.

\section{Ion Beam-induced Amorphization And Track Size}

The effective size of damaged zones formed from ion tracks initiated by individual bombarding ions can be estimated by numerical simulation compared with the dynamics of damage profiles measured by RBS and ellipsometry. ${ }^{55}$ In this case the numerical simulation assumes both a track size and a damage profile, the latter being measured by RBS. The evolution of damage was measured and calculated as a function 
of ion fluence, i.e., as snapshots of the process in time. In case of ellipsometry the analysis of the vanishing absorption features ${ }^{56}$ was enough to determine the dynamics of amorphization and the track size. ${ }^{55}$

A much simpler assumption is to take into account that the ions impinging at random positions of the surface satisfy the Poisson statistics, i.e. the probability that the next ion finds a non-damaged position is proportional to the area of the surface that has not yet been amorphized. ${ }^{56}$ Fig. $6(\mathrm{~A})$ shows the fit considering Poisson statistics for the evolution of $f_{a}$, i.e., the amorphous i-a-Ge fraction. The red line corresponds to the Poisson $\lambda$ parameter value of $\lambda \approx 11$ (computed in fluence units of $1 \cdot 10^{12} \mathrm{~cm}^{-2}$ ), and from $\lambda$ a corresponding track diameter of $2.5 \mathrm{~nm}$ (track area of $5 \mathrm{~nm}^{2}$ ) can be estimated. In the calculation it was considered that $\lambda=n p$, where $n$ is the total number of possible individual events (total number of different independent positions for incoming ions within a window of $1 \mathrm{~cm}^{2}$, i.e., the inverse of the track size, $\left(R_{T}^{2} \pi\right)^{-1}$, with $R_{T}$ being the track radius), and $p$ is the probability to find undamaged region by an incoming ion, for which the center value of $p=f_{a}=1 / 2$ was considered.

To ascertain more details about the progress of implantation-induced damage accumulation, we applied the direct-impact, defect-stimulated (D-I/D-S) amorphization model to reproduce the change in the amorphous fraction i-a-Ge vs. the applied ion fluence. Previously, this model has been successfully applied to describe the behavior of $\mathrm{Ge}^{46}$ and other semiconductor materials ${ }^{57}$ exposed to ion irradiation. In the D-I/D-S model, amorphous nuclei are directly produced in the core of a cascade (homogeneous amorphization) and irradiation-induced point defects and/or subsequently implanted ions stimulate further amorphization at the crystalline-amorphous interfaces (heterogeneous amorphization). If the probability for stimulated amorphization is taken as $f_{a}\left(1-f_{a}\right)$ then the differential change in $f_{a}$, due to an infinitezimal fluence, $d D$, can be written as:

$d f_{a} / d D=\sigma_{a}\left(1-f_{a}\right)+\sigma_{s} f_{a}\left(1-f_{a}\right)(1)$

where $\sigma_{a}$ is the direct-impact amorphization cross-section, and $\sigma_{s}$ is the effective cross-section for stimulated amorphization ${ }^{57}$. The fit for $f_{a}$ can also be seen in Fig. 6A with corresponding values of $\sigma_{a}=$ $0.6 \mathrm{~nm}^{2}$ and $\sigma_{s}=40 \mathrm{~nm}^{2}$, respectively. Note that, while the value of $\sigma_{a}=0.6 \mathrm{~nm}^{2}$ is comparable to an effective cross-section for defect formation, $\sigma_{\text {eff }} \approx 0.5-1 \mathrm{~nm}^{2}$ derived from SRIM vacancy profiles, the cross-section $\sigma_{s}=40 \mathrm{~nm}^{2}$ is much larger. That is, the overall damage formed in cascade processes initiated by one impinging ion is several times higher than predicted by SRIM. This result is consistent with molecular dynamics (MD) simulations performed for $5 \mathrm{keV} \mathrm{Sb}^{+}$bombardment into Ge, showing that a large number of defects can be formed in hot collision cascades, and most of them is contained in larger defect clusters, which can be thermodynamically more stable than single point defects ${ }^{58}$. The probability to form such complex defect structure is much higher for heavy $\mathrm{Sb}^{+}$ions than, e.g., for lighter $\mathrm{B}^{+}$or $\mathrm{Si}^{+}$projectiles ${ }^{50,58}$. For $5-\mathrm{keV} \mathrm{Sb}^{+}$bombardment $\mathrm{MD}$ simulations show that the total number of atomic displacements (about 2000/ion) is about 20 times higher than predicted by SRIM (about 
100/ion). This result is in agreement with our observations based on the D-I/D-S amorphization model. In general, the high damage cross-section for heterogeneous amorphization in $\mathrm{Sb}^{+}$-implanted Ge suggests its tendency for significant local atomic transport under these conditions that can be a prerequisite to initiate a spatial reorganization process and the formation of voids at higher ion fluences.

The different cross-sections for damage formation, obtained from Poisson statistics and the D-I/D-S model are due to the distinct basic assumptions applied. Note that the cross-section value given by the geometrical concept-based Poisson function falls between the values of $\sigma_{a}$ and $\sigma_{s}$ provided by the D-I/D$S$ model, which combines contributions to $f_{a}$ from both homogeneous and heterogeneous amorphization. In the D-I/D-S model the effect of the lower cross-section $\sigma_{a}$ is compensated with the higher cross-section $\sigma_{s}$ to reproduce the shape of the amorphous fraction curve, $f_{a}$.

Our derived cross-sections for direct-impact, and heterogeneous amorphization differ from the values found for $3 \mathrm{MeV} \mathrm{I}$ ion irradiation, where $\sigma_{a}=9 \mathrm{~nm}^{2}$ and $\sigma_{s}=20 \mathrm{~nm}^{2}$ were reported ${ }^{46}$. The reason for the differences can be explained considering the dissimilar conditions for the lower energy $\mathrm{Sb}^{+}$and the high energy $\mathrm{I}^{+}$irradiation. The electronic energy deposition which is associated with temperature increase and local target melting is about 4 times higher for the $\mathrm{I}^{+}$irradiation, as predicted by SRIM, and therefore the probability to directly form larger amorphous clusters via local melting and fast cooling can be increased compared to our $\mathrm{Sb}^{+}$implantation. On the other hand, the higher local temperature may be accompanied by different in-situ defect formation, annealing, cluster formation and diffusion kinetics of defects for $3 \mathrm{MeV} \mathrm{I}^{+}$compared to $200 \mathrm{keV} \mathrm{Sb}^{+}$. It is worth noting that in Ref. ${ }^{46}$ the amorphous Ge fraction has been extracted from ex-situ RBS/C measurements while in this work these data were obtained from in-situ SE spectra.

The effective size of the ion tracks found in this work applying the D-I/D-S amorphization model for Ge is about four times the size of tracks of Xe ions implanted in SiC at an energy of $100 \mathrm{keV}^{55}$, and 3-4 times the size of excitons in GaAs created by the implantation of As ions at an energy of $270 \mathrm{keV} .{ }^{56}$ However, the derived damage track size is much smaller than the exciton diameter of about $50 \mathrm{~nm}$ in $\mathrm{Ge}^{13}$. Differences in track sizes may be due to distinct materials properties, like displacement energy, collision cascade kinetics and relaxation, as well as thermal transport features, of the targets exposed to irradiation.

\section{Mechanisms Of Damage And Void Formation}

The temporal change of the ellipsometric model parameters is shown in Fig. 6B, using 2 layers on intact Ge substrate. Both layers contain c-Ge, i-a-Ge $\left(f_{a}\right)$ and void $\left(f_{v}\right)$ phases. It is important to emphasize that the 'stripes' of volume fractions in Fig. 6B are not vertically separated, in the optical model it is a uniform mixture of the indicated components within the layer, in fractions represented by the widths of the 'stripes'. The volume fraction of void, $f_{v}$, has previously been estimated by the expansion of the sample ${ }^{61}$ 
measured by Talystep or by the analysis of TEM images. ${ }^{44}$ The void parameter $f_{\mathrm{v}}$ from the ellipsometry fit was measured real time during irradiation. The approximate accuracy of the determination of $f_{v}$ was a few percent, at a time resolution of $3 \mathrm{~s}$. Effective thicknesses of i-a-Ge $\left(d_{a}\right)$ and void $\left(d_{v}\right)$ are shown in Fig. $6 \mathrm{C}$. Note, that both i-a-Ge and voids distribute in the modified depth continuously.

After starting the irradiation (temporal position of $\approx 60 \mathrm{~s}$ ), the thickness of the surface layer increases to a value of $\approx 50 \mathrm{~nm}$ within less than 10 seconds. Note that the penetration depth of light in i-a-Ge in the used wavelength range is a couple of times $10 \mathrm{~nm}$, which means that the saturation of $d_{a}$ may be caused by the limited penetration of light.

Based on the paper written by Kaiser et al. ${ }^{44}$, the critical fluence of void formation for Sb implantation into $\mathrm{Ge}$ is between $3 \cdot 10^{14}$ and $5 \cdot 10^{14} \mathrm{~cm}^{-2}$. These values were comparable to that found for Ge selfimplantation $\left(2 \cdot 10^{15} \mathrm{~cm}^{-2}\right) \cdot{ }^{45}$ In our experiment, the total fluence was $1 \cdot 10^{16} \mathrm{~cm}^{-2}$, which results in void formation at a fluence of $1 \cdot 10^{15} \mathrm{~cm}^{-2}$ measured at a high accuracy using the in-situ SE method (see Region III in Fig. 4 that can also be identified in Figs. 6B and 6C).

The proposed explanations of void formation in the literature range from sputtering and redeposition 22,62 through thermal spikes ${ }^{63}$ to clustering of vacancies and diffusion of interstitials. ${ }^{40,64}$ It has also been shown by different authors that the void formation obeys mass conservation, and the sputtering effect can be ruled out, ${ }^{22,44,65}$ also proved by the use of a capping layer, ${ }^{66}$ by molecular dynamic simulations ${ }^{62}$ and by scanning tunneling microscopy (STM) measurements. ${ }^{63}$

Formation of columnar voids of $20-40 \mathrm{~nm}$ in diameter in Ge have been reported by many authors for different experimental (preparation) conditions. ${ }^{22,61,67}$ It was found by high-energy heavy ion bombardment that the voids are formed by the agglomeration of vacancies, and a critical defect production rate is necessary to initiate the formation of the sponge-like structure, ${ }^{65}$ It was found feasible already in the early investigation of Appleton et al. that the primary cause of void formation is the high mobility of defects. ${ }^{67}$ This was underlined by the fact that implantation of $280-\mathrm{keV} B i$ into Ge at liquid nitrogen (LN) temperature did not lead to void formation, whereas annealing and high-temperature ion implantation leads to the decrease of dopant retainment, ${ }^{67}$ also pointing out the gettering effect of voids. ${ }^{68}$

In case of self-implantation at the energy of $500 \mathrm{keV}$ flattened vacancy agglomerates of $10.6 \mathrm{~nm}$ average size are formed at a fluence of $1 \cdot 10^{15} \mathrm{~cm}^{-2}$ at room temperature (RT). ${ }^{64}$ As shown in Fig. $6 \mathrm{~A}$, this is the temporal position in our measurement from which a pronounced growth of the damaged and porous layer starts. The study of Desnica-Fankovic et al. ${ }^{64}$ also shows that the size of these agglomerates grows to $17 \mathrm{~nm}$ at the fluence of $3 \cdot 10^{15} \mathrm{~cm}^{-2}$, and their shape becomes more spherical. At higher fluences, these nanoclusters agglomerate into larger voids of a broad size distribution, which completely dominates at the fluence of $3 \cdot 10^{16} \mathrm{~cm}^{-2}$. The thermal energy in the RT-implanted samples is large 
enough for the diffusion and restructuring of defects clustering the vacancies into voids and finally causing porosity - a feature that lacks in the case of implantation at LN temperature. ${ }^{64}$

The voids remain stable during annealing, which restricts shallow junction formation by the implantation of heavy elements. ${ }^{69}$ The void formation is the reason of finding unintentional $\mathrm{O}$ and $\mathrm{C}$ impurities by ion scattering measurement after removing the samples from vacuum. ${ }^{67}$ This structure can, however, be used for gettering as well. ${ }^{65,68}$ The structure created by the implantation of Sb into Ge can also be reproduced by other elements if the fluence and the temperature is high enough. ${ }^{40,66}$ Therefore, in device fabrication, lighter elements are used as dopants. ${ }^{69} \mathrm{~B}$ (with Ge pre-amorphization) and $\mathrm{P}$ (with selfamorphization) can be used as doping species with subsequent low-temperature annealing to achieve substitutional, electrically active positions of the dopants.

A detailed picture of the void formation mechanisms was shown by Nitta et al. for GaSb implanted by $\mathrm{Sn}^{+} .59,60$ Voids are formed by the migration of interstitials in the first stage of the implantation (Figs. 6D and $6 \mathrm{E}$, as well as region II in $6 \mathrm{~B}$ ). The interstitials are not stable at RT. ${ }^{70}$ Those that survive the annihilation process can migrate to the bottom of walls. In this model the walls develop by the aggregated interstitials (region III in Fig. 6B), while the voids by the vacancies migrating to the bottom of existing voids (Fig. 6F). This model does not explain the driving force for the interstitials to aggregate at the bottom of the walls. A plausible background to this phenomenon might be the different depth profiles of interstitials under the voids and the walls between the voids (due to the different amounts of materials above a certain position in depth depending on the amount of void in the path of the penetrating ion), which results in a lateral gradient of interstitial concentration in the lattice.

In the final stage the voids burst to the surface at higher fluencies, as shown in Fig. 6G. These processes are confirmed by the present results which show that the volume fraction of voids is larger in the embedded layers, and the void fraction in the surface layer still increases at the end of the process (Fig. 6B). Note that the damage depth range at the initial stage is consistent with the SRIM ion range calculation shown in Fig. 3B. Note that Fig. 6A covers approximately 90 minutes of measurement with $3 \mathrm{~s}$ steps, i.e. almost 2000 individually fitted spectra. The noise at the edge of the stripes represents the approximate uncertainty of the measurement (a few nanometers and a few percent for the thicknesses and the volume fractions, respectively). The two homogeneous composite layers are a simplification of a depth profile that may in the future be better approximated by an analytical gradient profile, which however, due to the presence of voids, might be more complex that those used for simple unperturbed Gaussian damage profiles. ${ }^{24,26}$

\section{Conclusions}

We developed an in-situ method for controlling the dynamics of structural damage accumulation during ion-implantation. The real-time (high temporal resolution) in-situ SE measurement combined with continuous determination of parameters on the evolution based on an appropriate optical model can help 
to avoid (detect) adverse void formation and for controlled evolution of subsurface nanocavities or cellular surface texture which, once formed, cannot be removed via annealing. The in-situ data also enable a more detailed insight into the dynamics of processes and provides quantitative data such as the size of tracks created by the implanted ions or the volume fraction of phases.

\section{Materials And Methods}

\section{Sample preparation}

Ge wafer from Umicore (orientation (100), resistivity of approx. $0.4 \Omega \cdot \mathrm{cm}$ ) was cleaned in diluted HF and rinsed in deionized (DI) water. After cutting into small rectangular pieces, the samples were rinsed again in $\mathrm{DI}$ and dried in $\mathrm{N}$ gas. The $200-\mathrm{keV} \mathrm{Sb}^{+}$ions were implanted into a c-Ge piece at a fluence of $1 \cdot 10^{16}$ $\mathrm{cm}^{-2}$ and ion flux of $2.1 \cdot 10^{12} \mathrm{~cm}^{-2} \mathrm{~s}^{-1}$.

\section{Measurement And Modeling By Se}

For ex-situ ellipsometric characterization, an M-2000DI (Institute of Technical Physics and Materials Science, Centre for Energy Research, Budapest, Hungary) and an M-2000F (Department of Optics and Quantum Electronics, University of Szeged, Hungary) rotating compensator spectroscopic ellipsometer was used. The M-88 spectroscopic ellipsometer attached to the implantation chamber was operated in the wavelength range from 367 to $746 \mathrm{~nm}$.

SE measures spectra of $\Psi$ and $\Delta$, that describes the complex reflectance ratio of $\rho=r_{p} / r_{s}=\tan (\Psi) \cdot \exp (i \Delta)$, where $r_{p}$ and $r_{s}$ denote the complex reflection ratio of light polarized parallel and perpendicular to the plane of incidence, respectively. ${ }^{32,71}$ The response of the sample is calculated using the transfer matrix method based on stratified media with modeled dispersion and thicknesses. The parameters are searched in the global parameter space, then fitted using the Levenberg-Marquardt gradient method to find the smallest discrepancy between the simulated and measured $\Psi$ and $\Delta$ spectra. The quality of the fit is measured by the root mean square difference between those spectra.

For the construction of optical models for evaluation of the SE spectra we need to determine the reference dielectric function of ion implantation amorphized $\mathrm{Ge}$. The reference amorphous layer has been created by two step amorphization ( $120 \mathrm{keV} \mathrm{Al}{ }^{+} 1 \cdot 10^{16}$ atom $/ \mathrm{cm}^{2}$ and $300 \mathrm{keV} \mathrm{Al}{ }^{+} 1 \cdot 10^{16}$ atom $\left./ \mathrm{cm}^{2}\right)$. The spectra obtained from multiple-angles-of-incidence (MAl) spectroellipsometric measurements were evaluated using a two-layer optical model. The WVASE32, CompleteEASE 6.41 as well as our selfdeveloped software were used for the evaluation of the measured spectra. From the results of the evaluation reference dielectric data has been created and published before ${ }^{23}$ for ion-implantation amorphized Ge. The complex dielectric function of the amorphized Ge layer was described in this work by the Tauc-Lorentz dispersion relation using the above reference for the initial parameter set, fitting only the energy position of the Lorentz oscillator for i-a-Ge. The mean square error of the fit was between 10-20 
over the whole process. The typical uncertainty of the determination of layer thicknesses and volume fractions was a few nanometers and a few percent, respectively.

\section{Complementary Characterization Methods}

The optical measurements were completed by scanning electron microscopy (SEM) and ion beam analysis. The implantation induced disorder was investigated by RBS combined with channeling. The RBS analysis was performed using the Hungarian lon-beam Physics Platform 5 MV Van de Graaff accelerator at the Institute for Particle and Nuclear Physics, Wigner Research Centre for Physics in Budapest.

\section{Declarations}

\section{Acknowledgements}

The authors are grateful for financial support from the OTKA K131515 and K129009 projects.

\section{Author contributions statement}

Measurements and evaluations: TL, ZZ, AN, BK, AR, NQK, ES, EK, PK, JW, EA, ZT JB, PP, MF; writing and visualization: TL, ZZ, BK, AR, PP, MF, IB, JG; resources: TL, ES, PP, MF, IB, JG; conceptualization and supervision: TL, PP, MF; coordination: TL, PP, MF; review and editing: TL, ZZ, PP, MF, IB, JG.

\section{Competing interest statement}

The authors declare that they have no known competing financial interests or personal relationships that could have appeared to influence the work reported in this paper.

\section{References}

1. Cosentino, S. et al. Room-temperature efficient light detection by amorphous Ge quantum wells. Nanoscale Res. Lett. 8, 128 (2013).

2. Colace, L. et al. Ge on Si p-i-n photodiodes operating at 10Gbit/s. Appl. Phys. Lett. 88, 101111 (2006).

3. J. Opt. Soc. Am. B, JOSAB 30, 838-842 (2013).

4. Carletti, L. et al. Mid-infrared nonlinear optical response of Si-Ge waveguides with ultra-short optical pulses. Opt. Express. 23, 32202 (2015).

5. Bean, J. C. Silicon-based semiconductor heterostructures: column IV bandgap engineering. Proceedings of the IEEE 80, 571-587 (1992).

6. Lohner, T. et al. Refractive Index Variation of Magnetron-Sputtered a-Si1 - xGex by "One-Sample Concept" Combinatory. Applied Sciences. 8, 826 (2018). 
7. Hayashi, S., Ito, M. \& Kanamori, H. Raman study of gas-evaporated germanium microcrystals. Solid State Commun. 44, 75-79 (1982).

8. Canham, L. T. Silicon quantum wire array fabrication by electrochemical and chemical dissolution of wafers. Appl. Phys. Lett. 57, 1046-1048 (1990).

9. Agocs, E. et al. Optical and structural characterization of Ge clusters embedded in ZrO2. Appl. Surf. Sci. 421, 283-288 (2017).

10. Lehninger, D. et al. Ge Nanostructures Embedded in ZrO2 Dielectric Films for Nonvolatile Memory Applications. ECS Transactions. 66, 203-212 (2015).

11. Serényi, M. et al. Studies on the RF sputtered amorphous SiGe thin films. Inorg Mater. 42, 3-6 (2006).

12. Kalas, B. et al. Micro-combinatorial sampling of the optical properties of hydrogenated amorphous $\mathrm{Si}_{1-\mathrm{x}} \mathrm{Ge}_{\mathrm{x}}$ for the entire range of compositions towards a database for optoelectronics. Scientific Reports under publication, 18.

13. Barbagiovanni, E. G., Lockwood, D. J., Simpson, P. J. \& Goncharova, L. V. Quantum confinement in Si and Ge nanostructures: Theory and experiment. Applied Physics Reviews. 1, 011302 (2014).

14. Al-Mahasneh, A. A., Attar, A. \& Shahin, I. S. Spectroscopic ellipsometry of single and multilayer amorphous germanium/aluminum thin film systems. Opt. Commun. 220, 129-135 (2003).

15. Rafla-Yuan, H., Rancourt, J. D. \& Cumbo, M. J. Ellipsometric study of thermally evaporated germanium thin film. Appl. Opt. 36, 6360-6363 (1997).

16. Ziegler, J. F. Ion Implantation: Science and Technology (Ion Implantation Technology Co., 1996).

17. Lohner, T. et al. Comparative study of ion implantation caused anomalous surface damage in silicon studied by spectroscopic ellipsometry and Rutherford backscattering spectrometry. Nuclear Instruments and Methods in Physics Research Section B: Beam Interactions with Materials and Atoms. 147, 90-95 (1999).

18. Battistig, G. et al. Effect of ion current density on damage in Al ion implanted SiC. Nuclear Instruments and Methods in Physics Research Section B: Beam Interactions with Materials and Atoms. 219-220, 652-655 (2004).

19. Aspnes, D. E. \& Studna, A. A. An investigation of ion-bombarded and annealed ã $€^{\wedge} 111 a ̃ € \%$ surfaces of Ge by spectroscopic ellipsometry. Surf. Sci. 96, 294-306 (1980).

20. Dekker, J., Zandvliet, H. J. W. \& van Silfhout, A. Low energy ion bombardment on c-Ge surfaces. Vacuum. 41, 1690-1691 (1990).

21. Zolnai, Z. et al. Damage accumulation in nitrogen implanted 6H-SiC: Dependence on the direction of ion incidence and on the ion fluence. Journal of Applied Physics. 101, 023502 (2007).

22. Wilson, I. H. The effects of self-ion bombardment (30-500 keV) on the surface topography of singlecrystal germanium. J. Appl. Phys. 53, 9 (1982).

23. Lohner, T. et al. Determination of the Complex Dielectric Function of Ion-Implanted Amorphous Germanium by Spectroscopic Ellipsometry. Coatings. 10, 480 (2020). 
24. Fried, M., Lohner, T., Aarnink, W. M., Hanekamp, L. J. \& van Silfhout, A. Nondestructive determination of damage depth profiles in ion-implanted semiconductors by spectroscopic ellipsometry using different optical models. Journal of Applied Physics. 71, 2835-2843 (1992).

25. Szilágyi, E. et al. Oxidation of SiC investigated by ellipsometry and Rutherford backscattering spectrometry. Journal of Applied Physics. 104, 014903 (2008).

26. Petrik, P. et al. Ellipsometric characterization of damage profiles using an advanced optical model. Journal of Applied Physics. 93, 1987-1990 (2003).

27. Petrik, P. et al. Optical models for ultrathin oxides on Si- and C-terminated faces of thermally oxidized SiC. Journal of Applied Physics. 106, 123506 (2009).

28. Petrik, P., Racz, A. S. \& Menyhard, M. Complementary physicochemical analysis by ellipsometry and Auger spectroscopy of nano-sized protective coating layers. Appl. Surf. Sci. 534, 147593 (2020).

29. Adachi, S. Optical Constants of Crystalline and Amorphous Semiconductors: Numerical Data and Graphical Information. (Springer Science \& Business Media 1999).

30. Wei, P., Xu, Y., Nagata, S., Narumi, K. \& Naramoto, H. Structure and optical properties of germanium implanted with carbon ions. Nuclear Instruments and Methods in Physics Research Section B: Beam Interactions with Materials and Atoms. 206, 233-236 (2003).

31. Lehninger, D. et al. Charge trapping of Ge-nanocrystals embedded in TaZrO x dielectric films. Appl. Phys. Lett. 106, 023116 (2015).

32. Fujiwara, H. Spectroscopic Ellipsometry: Principles and Applications. 369 (2007). doi:10.1002/9780470060193.

33. Petrik, P. Parameterization of the dielectric function of semiconductor nanocrystals. Physica $B$ : Condensed Matter. 453, 2-7 (2014).

34. Fried, M., Lohner, T., Aarnink, W. A. M., Hanekamp, L. J. \& van Silfhout, A. Determination of complex dielectric functions of ion implanted and implanted-annealed amorphous silicon by spectroscopic ellipsometry. Journal of Applied Physics. 71, 5260-5262 (1992).

35. Collins, R. W., An, I., Chen, C., Ferlauto, A. S. \& Zapien, J. A. Advances in multichannel ellipsometric techniques for in-situ and real-time characterization of thin films. Thin Solid Films. 469-470, 38-46 (2004).

36. Hu, Y. Z. et al. Studies of hydrogen ion beam cleaning of silicon dioxide from silicon using in situ spectroscopic ellipsometry and x-ray photoelectron spectroscopy. Appl. Phys. Lett. 58, 589-591 (1991).

37. Hu, Y. Z., Andrews, J. W., Li, M. \& Irene, E. A. In situ spectroscopic ellipsometric investigation of argon ion bombardment of single-crystal silicon and silicon dioxide films. Nuclear Instruments and

Methods in Physics Research Section B: Beam Interactions with Materials and Atoms. 59-60, 76-79 (1991).

38. Petrik, P. et al. Ellipsometric characterization of shallow damage profiles created by Xe-implantation into silicon. in Ion Implantation Technology. 2002. Proceedings of the 14th International Conference on 601-604 (IEEE, 2002). doi:10.1109/IIT.2002.1258077. 
39. Petrik, P. et al. Ion implantation induced disorder in single-crystal and sputter-deposited polycrystalline $\mathrm{CdTe}$ characterized by ellipsometry and backscattering spectrometry. phys. stat. sol. (c). 5, 1358-1361 (2008).

40. Holland, O. W., Appleton, B. R. \& Narayan, J. Ion implantation damage and annealing in germanium. Journal of Applied Physics. 54, 2295-2301 (1983).

41. Petrik, P. et al. Depth distribution of disorder and cavities in high dose helium implanted silicon characterized by spectroscopic ellipsometry. Thin Solid Films. 455-456, 344-348 (2004).

42. Petrik, P. et al. Ion implantation-caused damage in SiC measured by spectroscopic ellipsometry. Thin Solid Films. 455-456, 239-243 (2004).

43. Khaibullin, I. B. et al. Effect of Heavy Ion Implantation and Laser Annealing on the Structural Properties of Germanium. phys. stat. sol. (a). 94, 371-377 (1986).

44. Kaiser, R. J. et al. Honeycomb voids due to ion implantation in germanium. Thin Solid Films. 518, 2323-2325 (2010).

45. Darby, B. L. et al. Insights for void formation in ion-implanted Ge. Thin Solid Films. 519, 5962-5965 (2011).

46. Steinbach, T., Wernecke, J., Kluth, P., Ridgway, M. C. \& Wesch, W. Structural modifications of lowenergy heavy-ion irradiated germanium. Phys. Rev. B. 84, 104108 (2011).

47. Steinbach, T. \& Wesch, W. Porous structure formation in ion irradiated germanium. Nuclear Instruments and Methods in Physics Research Section B: Beam Interactions with Materials and Atoms. 319, 112-116 (2014).

48. Pászti, F., Manuaba, A., Hajdu, C. \& Melo, A. A. \& Da Silva, M. F. Current measurement on MeV energy ion beams. Nuclear Inst. and Methods in Physics Research, B. 47, 187-192 (1990).

49. Ziegler, J. F., Ziegler, M. D. \& Biersack, J. P. SRIM - The stopping and range of ions in matter (2010). Nuclear Instruments and Methods in Physics Research Section B: Beam Interactions with Materials and Atoms. 268, 1818-1823 (2010).

50. Gomez-Selles, J. L., Claverie, A., Sklenard, B. \& Benistant, F. \& Martin-Bragado, I. Atomistic simulation of damage accumulation and amorphization in Ge. Journal of Applied Physics. 117, 055703 (2015).

51. Johs, B., Herzinger, C. M., Dinan, J. H., Cornfeld, A. \& Benson, J. D. Development of a parametric optical constant model for $\mathrm{Hg} 1 \mathrm{yxCd} \times \mathrm{Te}$ for control of composition by spectroscopic ellipsometry during MBE growth.6(1998).

52. Aspnes, D. E. Plasmonics and Effective-Medium Theory. in Ellipsometry at the Nanoscale (eds. Losurdo, M. \& Hingerl, K.)203-224(SpringerBerlin Heidelberg, 2013).

53. Lohner, T. et al. Refractive Index Variation of Magnetron-Sputtered a-Si1 - xGex by "One-Sample Concept" Combinatory. Applied Sciences. 8, 826 (2018).

54. Jellison, G. E. \& Modine, F. A. Parameterization of the optical functions of amorphous materials in the interband region. Appl. Phys. Lett. 69, 371-373 (1996). 
55. Petrik, P. et al. Characterization of damage structure in ion implanted SiC using high photon energy synchrotron ellipsometry. Thin Solid Films. 519, 2791-2794 (2011).

56. Kelso, S. M., Aspnes, D. E., Olson, C. G. \& Lynch, D. W. Optical determination of exciton sizes in semiconductors. Physica B + C. 117-118, 362-364 (1983).

57. Hecking, N., Heidemann, K. F. \& Te Kaat, E. Model of temperature dependent defect interaction and amorphization in crystalline silicon during ion irradiation. Nuclear Instruments and Methods in Physics Research Section B: Beam Interactions with Materials and Atoms. 15, 760-764 (1986).

58. López, P., Pelaz, L., Santos, I., Marqués, L. A. \& Aboy, M. Molecular dynamics simulations of damage production by thermal spikes in Ge. Journal of Applied Physics. 111, 033519 (2012).

59. Nitta, N. et al. Anomalous Defect Structure Formed on GaSb Surface by Low Temperature Sn lonImplantation and Its Formation Mechanism. J.Japan Inst.Metals. 64, 1141-1147 (2000).

60. Nitta, N., Taniwaki, M., Hayashi, Y. \& Yoshiie, T. Formation of cellular defect structure on GaSb ionimplanted at low temperature. Journal of Applied Physics. 92, 1799-1802 (2002).

61. Destefanis, G. L. \& Gailliard, J. P. Very efficient void formation in ion implanted InSb. Appl. Phys. Lett. 36, 40-42 (1980).

62. Bellon, P. et al. Surface damage produced by $20 \mathrm{keV} \mathrm{Ga} \mathrm{bombardment} \mathrm{of} \mathrm{Ge(001).} \mathrm{Surf.} \mathrm{Sci.} \mathrm{339,}$ 135-141 (1995).

63. Kim, J. C., Cahill, D. G. \& Averback, R. S. Surface defects created by $20 \mathrm{keV}$ Xe ion irradiation of Ge(1 11). Surf. Sci. 574, 175-180 (2005).

64. Desnica-Franković, I. D. et al. GISAXS studies of structural modifications in ion-beam amorphized Ge. Nuclear Instruments and Methods in Physics Research, Section B: Beam Interactions with Materials and Atoms. 249, 114-117 (2006).

65. Huber, H. et al. Void formation in Ge induced by high energy heavy ion irradiation. Nuclear Instruments and Methods in Physics Research Section B: Beam Interactions with Materials and Atoms. 122, 542-546 (1997).

66. Janssens, T. et al. Heavy ion implantation in Ge: Dramatic radiation induced morphology in Ge. J. Vac. Sci. Technol. B. 24, 510 (2006).

67. Appleton, B. R. et al. Characterization of damage in ion implanted Ge. Appl. Phys. Lett. 41, 711-712 (1982).

68. Petrik, P. et al. Optical models for cavity profiles in high-dose helium-implanted and annealed silicon measured by ellipsometry. Journal of Applied Physics. 97, 123514 (2005).

69. Satta, A. et al. Shallow Junction Ion Implantation in Ge and Associated Defect Control. J. Electrochem. Soc. 153, G229 (2006).

70. Koffel, S., Scheiblin, P., Claverie, A. \& Benassayag, G. Amorphization kinetics of germanium during ion implantation. Journal of Applied Physics. 105, 013528 (2009).

71. Collins, R. W. \& Ferlauto, A. S. Optical Physics of Materials. in Handbook of ellipsometry (eds. Irene, E. G. \& Tomkins, H. G.) (William Andrew, 2005). 